\title{
Acute alcohol administration dampens threat-related activation in the central extended amygdala
}

Juyoen Hur ${ }^{1 *}$

Claire M. Kaplan ${ }^{1 *}$

Jason F. Smith ${ }^{1 *}$

Daniel E. Bradford 4

Andrew S. Fox ${ }^{5,6}$

John J. Curtin ${ }^{4}$

Alexander J. Shackman ${ }^{1-3}$

${ }^{1}$ Department of Psychology, ${ }^{2}$ Neuroscience and Cognitive Science Program, and ${ }^{3}$ Maryland Neuroimaging Center, University of Maryland, College Park, MD 20742 USA. ${ }^{4}$ Department of Psychology, University of Wisconsin-Madison, 1202 West Johnson Street, Madison, WI 53706 USA. ${ }^{5}$ Department of Psychology and ${ }^{6}$ California National Primate Research Center, University of California, Davis, CA 95616 USA

* contributed equally

Words: 3,336

Figures: 2

Tables: 2

SOM: 1 document detailing supplementary methods and results

Keywords: affective neuroscience, anxiolytic, bed nucleus of the stria terminalis (BST/BNST), central extended amygdala (EAc), ethyl alcohol, fear and anxiety, functional MRI (fMRI)

\section{Address Correspondence to:}

Alexander J. Shackman (shackman@umd.edu)

Biology-Psychology Building, University of Maryland, College Park MD 20742 USA 


\section{ABSTRACT (256 words)}

Alcohol abuse is common, imposes a staggering burden on public health, and is challenging to treat, underscoring the need to develop a deeper understanding of the underlying neurobiology. When administered acutely, ethyl alcohol reduces threat reactivity in humans and other animals, and there is growing evidence that threat-dampening and related negative reinforcement mechanisms support the etiology and recurrence of alcohol and other kinds of substance misuse. Converging lines of evidence motivate the hypothesis that these effects are mediated by the central extended amygdala (EAc)—including the central nucleus of the amygdala (Ce) and bed nucleus of the stria terminalis (BST) - but the relevance of this circuitry to acute alcohol effects in humans remains poorly understood. Using a single-blind, randomizedgroups design, multiband imaging data were acquired from 49 social drinkers while they performed an fMRI-optimized emotionalfaces/places paradigm after consuming alcohol or placebo. Relative to placebo, alcohol significantly dampened reactivity to threat-related emotional faces in the BST. To rigorously assess potential regional differences in activation, data were extracted from anatomically defined Ce and BST regions-of-interest. Analyses revealed a similar pattern of dampening across the two regions. In short, alcohol acutely dampens reactivity to threat-related faces in humans and it does so similarly across the two major divisions of the EAc. These observations provide a framework for understanding the translational relevance of addiction models derived from work in rodents, inform on-going debates about the functional organization of the EAc, and set the stage for bi-directional translational models aimed at developing improved treatment strategies for alcohol abuse and other addictions. 


\section{INTRODUCTION}

Alcohol abuse is common (i.e., nearly three-quarters of Americans consumed some form of ethanol in the past year and, among them, $17.5 \%$ met criteria for an alcohol use disorder); contributes to a wide range of adverse social outcomes (e.g., crime); and imposes a substantial and growing burden on global public health and the economy ${ }^{1-3}$. Existing treatments are incompletely effective ${ }^{4}$, underscoring the urgency of developing a clearer understanding of the neural systems contributing to the development, maintenance, and recurrence of alcohol abuse ${ }^{5}$. When administered acutely, alcohol has anxiolytic properties in humans and rodents ${ }^{6-8}$, and there is clear evidence that threat- and stressdampening effects (i.e., negative reinforcement) contribute to the etiology and recurrence of alcohol misuse and abuse ${ }^{8,9}$. Yet, remarkably little is known about the neural circuitry underlying the threat-dampening effects of acute alcohol administration in humans.

Converging lines of mechanistic, anatomical, physiological, and pharmacological evidence highlight the potential importance of the central extended amygdala (EAc), including the central nucleus of the amygdala (Ce) and bed nucleus of the stria terminalis (BST). The EAc plays a critical role in assembling defensive responses to a range of threats ${ }^{10-13}$. Anatomically, the EAc is poised to govern vigilance and other aspects of fear and anxiety via dense mono- and poly-synaptic projections to downstream effector regions ${ }^{14,15}$. Imaging and lesion studies demonstrate that the EAc plays a key role in selecting and prioritizing the processing of 'threat-related' cues, such as fearful faces ${ }^{16}$ (see Supplementary Comment). EAc function co-varies with individual differences in anxious temperament and likely contributes to the development and maintenance of anxiety disorders 12,15-17. Conversely, acute administration of classic anxiolytics (e.g., diazepam, lorazepam) is associated with reduced reactivity of the dorsocaudal amygdala (in the region of the Ce) to threat-related faces ${ }^{18,19}$. 
Despite this progress, the relevance of the EAc to alcohol-induced threat-dampening in humans remains poorly understood. Although a few functional MRI (fMRI) studies have been reported (Table 1), with some suggesting that alcohol dampens amygdala reactivity to threat-related faces (i.e., fearful or angry expressions), they are limited by small samples $(\mathrm{Ns}<15)$ and coarse spatial resolution. Many relied on thresholding procedures that are now known to markedly inflate the risk of false discoveries ${ }^{20,21}$. Often, null results were interpreted as evidence of threatdampening ${ }^{22}$. None explicitly examined either the Ce or the BST ${ }^{23-26 . ~ A l l ~ r e l i e d ~ o n ~ r a n d o m i z e d ~ c r o s s-o v e r ~ d e s i g n s, ~ d e s p i t e ~ e v i d e n c e ~}$ questioning the retest reliability of amygdala activation e.g., ${ }^{27}$. In short, it remains unclear whether the Ce and BST are dampened by alcohol and, if so, whether they differ in their sensitivity. 
Table 1. The effects of acute alcohol administration on reactivity to threat-related faces in human imaging studies.

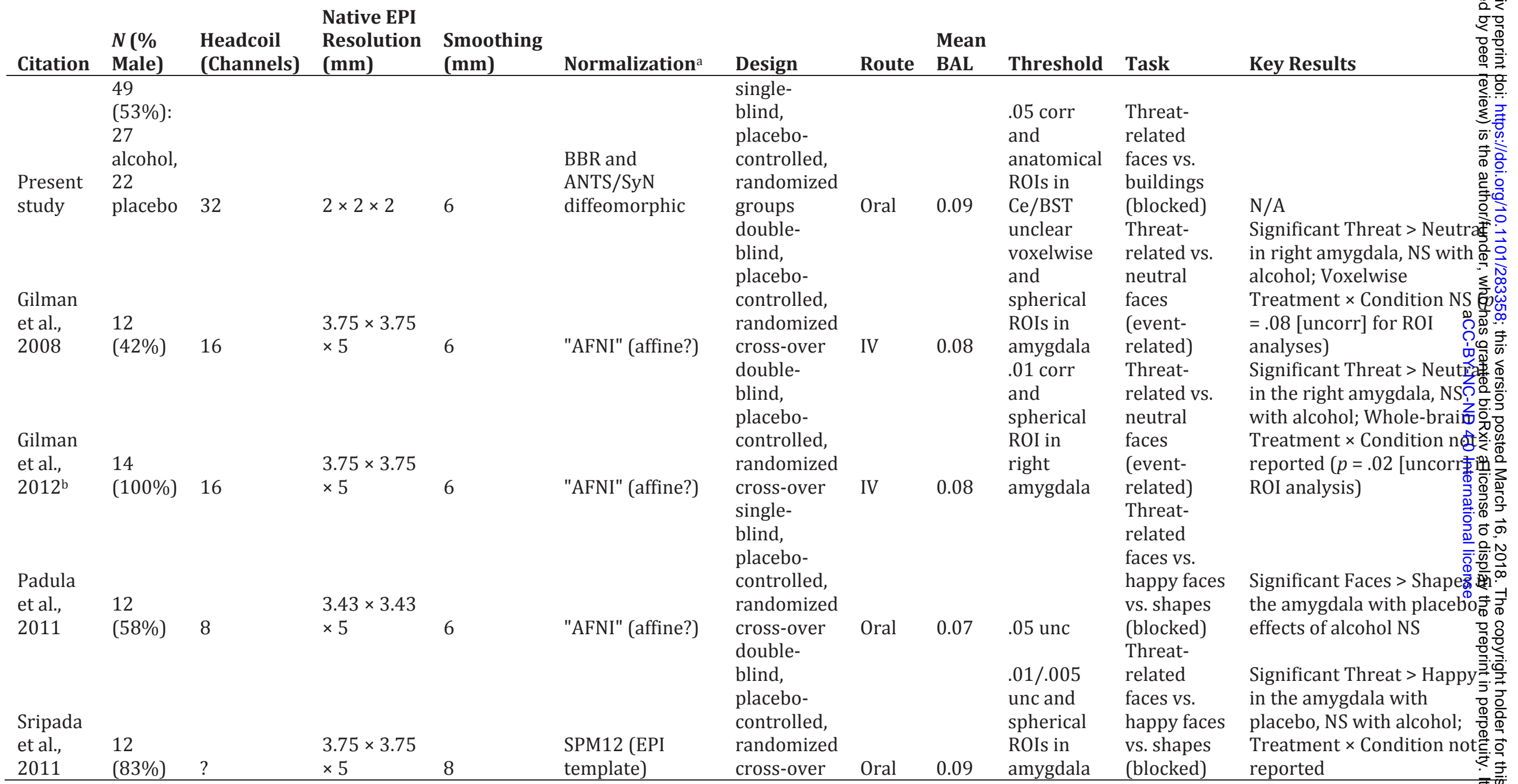

a Older normalization techniques (e.g., affine, EPI-to-EPI) introduce substantial spatial smoothing and registration error, which is a concern for work focused on small subcortical structures, such as the EAc. b Social drinker ('control') group. Abbreviations-BAL, blood alcohol level; BBR, boundary-based registration of the T1- and T2weighted images; Corr, corrected; IV, intravenous; N/A, not applicable; NS, not significant; Unc, uncorrected. 
Here, we used a novel combination of approaches to assess the influence of acute alcohol administration on reactivity to threat-related faces in the Ce and the BST. Using a single-blind, placebo-controlled, randomized-groups design (Table 2), fMRI data were acquired from 49 psychiatrically healthy social drinkers while they performed an fMRI-optimized emotional-faces/places paradigm after consuming an alcoholic or placebo beverage. Several methods enhanced precision, including a multiband pulse sequence and advanced co-registration and spatial normalization techniques ${ }^{28}$. Recently developed, anatomically defined Ce and BST regions-of-interest (ROIs) ${ }^{29,30}$ made it possible to directly compare the hypothesized threat-dampening effects of alcohol in the BST and the Ce for the first time. Understanding the role of the EAc in alcohol-induced threat-dampening is important. It is a necessary step to determining the translational relevance of addiction models derived from rodent models e.g., ${ }^{31}$. It would also provide insight into the EAc's role in social drinking and other kinds of substance use, inform on-going debates about the functional organization of the EAc 12,32,33, and guide the development of bi-directional translational models 12,34 aimed at developed improved treatment strategies 5,35 .

\section{METHOD}

Methods and materials are summarized below. Detailed descriptions are provided in the Supplement.

\section{Subjects}

Eighty-seven social drinkers (21-35 years old) were recruited from the community as part of a larger study. All reported an absence of substance, neurological, or psychiatric problems. Of these, 61 completed the emotional faces/places paradigm. Twelve subjects were excluded from analyses due to unusable anatomical data $(n=3)$, scanner problems $(n=1)$, incidental neurological findings ( $n=2)$, inadequate behavioral 
performance ( $>2 S D$ s below the mean; $n=3$ ), or excessive motion artifact (see below; $n=3$ ), yielding a final sample of 49 subjects (Table 2). Subjects provided informed written consent. Procedures were approved by the local Institutional Review Board.

Table 2. Demographic and descriptive variables.

\begin{tabular}{|c|c|c|c|c|}
\hline & Total & Placebo & Alcohol & Difference \\
\hline Sample size & 49 & 22 & 27 & $\mathrm{~N} / \mathrm{A}$ \\
\hline Mean Age in Years $(S D)$ & $22.4(2.5)$ & $22.1(1.4)$ & $22.6(3.1)$ & $t(47)=.69, p=0.50$ \\
\hline Gender: Female/Male & $23 / 26$ & $11 / 11$ & $12 / 15$ & $\chi^{2}=.15, p=0.8$ \\
\hline Mean BAL a $(S D)$ & $\mathrm{N} / \mathrm{A}$ & $0.00(0.00)$ & $0.09(0.02)$ & $t(47)=27.20, p<.001$ \\
\hline $\begin{array}{l}\text { Mean Subjective Estimate of } \\
\text { Number of Drinks Consumed } \\
\text { During the Study }(S D)\end{array}$ & $\mathrm{N} / \mathrm{A}$ & $2.07(1.09)^{\mathrm{b}}$ & $4.56(1.25)^{c}$ & $t(47)=7.32, p<.001$ \\
\hline $\begin{array}{l}\text { Mean Motion, Frame-to- } \\
\text { Frame Displacement }(S D)\end{array}$ & $.13(.03)$ & $.12(.03)$ & $.13(.03)$ & $t(47)=1.01, p=.32$ \\
\hline
\end{tabular}

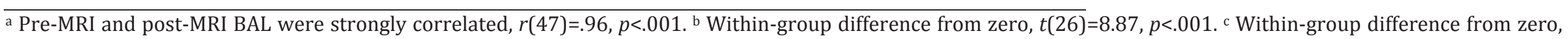
$t(26)=18.93, p<.001$.

\section{Overview and General Procedures}

Subjects abstained from alcohol and other substances for 24 hours and food/drink for 3 hours prior to the session. At the start of the session, subjects were randomly assigned (stratified by sex and race/ethnicity) to receive an alcoholic or placebo beverage, which was consumed just prior to scanning. Blood alcohol level (BAL) was assessed (Alcosensor IV Breathalyzer; Intoximeters Inc., St. Louis, MO) immediately before and after scanning. Subject status was continuously monitored using an MRI-compatible eye-tracker. At the end of the session, subjects estimated the number of standard alcoholic drinks they had consumed. 


\section{Alcohol/Placebo Procedures}

Well-established procedures were used for administering alcohol or placebo ${ }^{36,37}$. Consistent dosing was achieved using a formula that uses height, weight, age, and sex to produce a target BAL of $0.08 \%$ or $0.12 \%$ with an anticipated variance of $\pm 0.02 \% \sim 30$ minutes after the completion of beverage consumption ${ }^{38}$. This produced a unimodal BAL distribution (range: 0.06\% - 0.12\%; Table 2). Alcoholic beverages contained a mixture of juice and 100-proof vodka. To control absorption, subjects consumed 3 equal doses over 30 minutes. The placebo group received a similar beverage, with distilled water replacing the vodka. Subjects assigned to the alcohol (or placebo) group observed the experimenter pouring the vodka (or distilled water) from a vodka bottle. The placebo manipulation was reinforced by floating 3 ml of bitters and $3 \mathrm{ml}$ of vodka on the surface of the beverage and delivering a minute amount of aerosolized vodka to the rim of the beverage containers outside the subject's view. On average, subjects in the placebo group estimated that they consumed $\sim 2$ drinks, validating the manipulation (Table 1).

\section{Emotional-Faces/Places Paradigm}

Building on work by our group ${ }^{39}$ and others ${ }^{19,40}$, imaging data were acquired while subjects viewed alternating blocks of emotional faces ( 8 blocks) or places (9 blocks). Block length ( $16.3 \mathrm{~s})$ was optimized to detect between-condition differences ${ }^{41,42}$. To minimize habituation ${ }^{41-43}$, blocks consisted of 16 brief presentations of faces or places ( 1.02 s/image). During face blocks, subjects discriminated threat-related (i.e., fearful; 75\% trials) from neutral expressions (25\% trials) presented in a pseudorandomized order ${ }^{39}$. This design choice was aimed at reducing 
monotony and minimizing potential habituation to the fearful expressions ${ }^{43}$. During place blocks, subjects discriminated suburban residential residences (i.e., houses; 75\%) from urban commercial buildings (i.e., skyscrapers; 25\%). Face and place stimuli were adapted from prior work 44,45 .

\section{MRI Data Acquisition}

MRI data were acquired using a Siemens Magnetom TIM Trio 3 Tesla scanner (32-channel head-coil). Sagittal T1-weighted images were acquired using a MPRAGE sequence $(\mathrm{TR}=1,900 \mathrm{~ms}$; TE=2.32 ms; inversion time=900 ms; flip angle=9; sagittal slice thickness=0.9 mm; inplane $=0.449 \times 0.449 \mathrm{~mm}$; matrix $=512 \times 512$; field-of-view $=230 \times 230$ ). To enhance resolution, a multi-band sequence was used to collect a total of 286 oblique-axial EPI volumes during a single scan of the faces/places task (multiband acceleration=6; TR=1,000 ms; TE=39.4 ms; flip angle $=36.4^{\circ}$; slice thickness $=2.2 \mathrm{~mm}$, number of slices=60; in-plane resolution=2.1875×2.1875 mm; matrix=96 $\times 96$ ). To minimize susceptibility artifacts, images were collected in the oblique axial plane. Co-planar oblique-axial spin echo (SE) images were collected in opposing phase-encoding directions (TR=7,220 ms; TE=73 ms) to enable fieldmap correction.

\section{MRI Data Preprocessing}

MRI data were visually inspected before and after processing for quality assurance.

Anatomical Data Processing. Methods are similar to those described in other recent reports by our group $28,30$. T1 images were brainextracted ('skull-stripped') using a multi-tool approach ${ }^{30}$. Brain-extracted T1 images were normalized to the MNI152 template using the high- 
precision diffeomorphic approach implemented in $S y N^{46}$. The mean of the normalized T1 images is depicted in Supplementary Figure S1. FSL was used to create a fieldmap and undistorted SE image.

Functional Data Processing. The first 3 volumes of each EPI scan were removed. Remaining volumes were de-spiked and slice-time corrected using AFNI 47. For co-registration of the functional and anatomical images, an average EPI image was created. The average image was simultaneously co-registered with the corresponding T1-weighted image in native space and corrected for geometric distortions using the boundary-based registration method implemented in FSL and the previously created fieldmap, undistorted SE image, and T1 image. Spatial transformations were concatenated and applied to the functional data in a single step. The transformed images were re-sliced (2-mm ${ }^{3}$ ), smoothed (6-mm), and filtered (0.0078125-Hz high-pass). To assess residual motion artifact, the variance of volume-to-volume displacement of a selected voxel in the center of the brain $(x=5, y=34, z=28)$ was calculated using the motion-corrected EPI data. Subjects $(n=3)$ with extreme motion variance $(>2 S D$ s above the mean) were excluded from analyses.

fMRI data were modeled using SPM12 and in-house MATLAB code. The emotional-faces/places task was modeled using a boxcar function ${ }^{48}$. Block onsets were modeled using two additional event-related nuisance predictors. Predictors were convolved with a canonical hemodynamic response function. Additional nuisance variates included motion and physiological noise estimates. To attenuate physiological noise, white matter (WM) and cerebrospinal fluid (CSF) time-series were identified by thresholding the tissue prior images distributed with FSL. The EPI time-series was orthogonalized with respect to the first 3 right eigenvectors of the data covariance matrix from the WM and CSF compartments 
49. Reactivity to threat-related faces (i.e., the main effect of Condition: Emotional Faces vs. Places) was assessed using a voxelwise one-sample $t$ test controlling for mean-centered age and sex. The impact of alcohol administration was assessed using a voxelwise two-sample $t$ test controlling for mean-centered age and sex, equivalent to testing the Group (Alcohol vs. Placebo) $\times$ Condition (Emotional Faces vs. Places) interaction.

\section{Hypothesis Testing Strategy}

Alcohol-Dampening in the EAc. The first aim of the study was to test the hypothesized dampening effects of acute alcohol administration on EAc reactivity to threat-related faces. Accordingly, the Group $\times$ Condition interaction was thresholded at $p<.05$ familywise error (FWE) corrected for the extent of the EAc (Supplementary Figure S2; 1,205 voxels; 9,640 $\mathrm{mm}^{3}$ ). The EAc region-of-interest (ROI) encompassed the amygdala, substantia innominata/sublenticular extended amygdala (SI/SLEA), and BST bilaterally ${ }^{30,50}$. Significant clusters ( $p<.05$, wholebrain FWE corrected) outside the EAc are reported on an exploratory basis for voxelwise analyses of the Condition (Emotional Faces vs. Places) and Group $\times$ Condition effects.

Alcohol-Dampening: BST vs. Ce. The second major aim of our study was to test the differential sensitivity of the BST and the Ce-the two major sub-divisions of the EAc - to the hypothesized threat-dampening effects of alcohol. To do so in an unbiased manner, we extracted and averaged standardized contrast coefficients using anatomically defined, a priori ROIs ${ }^{21}$, as shown in Supplementary Figure S3. The BST was defined using the ROI of Theiss and colleagues (2016). The Ce was defined using the ROI of Tillman and colleagues (2018). A mixed-model general linear model was used to compare the impact of Group and Hemisphere on regional reactivity to threat-related faces. Significant 
interactions were decomposed using the appropriate tests of simple effects. The Group effect is reported using the Welch-Satterthwaite correction for unequal variances $\left(F_{W-S}\right)$.

\section{RESULTS}

\section{Behavior}

On average, subjects were highly accurate at performing the simple discrimination tasks $(M=86.8 \%, S D=7.9)$. Nevertheless, performance was $\sim 8 \%$ lower in the alcohol $(M=83.2 \%, S D=8.2)$ compared to the placebo group $\left(M=91.1 \%, S D=4.9 ; F_{W-S}(1,47)=15.98, p<.001\right)$, consistent with prior work 51 . Subjects were $\sim 4 \%$ more accurate when performing the places $(M=88.8 \%, S D=8.8)$ compared to the faces discrimination $(M=84.4 \%, S D=8.4 ; F(48)=22.37, p<.001)$, but the Group $\times$ Condition interaction was not reliable $(F(1,47)=.24, p=.63)$. As noted below and detailed in the Supplement, control analyses indicated that these modest differences in performance were not the primary determinant of alcohol-related differences in neural reactivity.

\section{The Dorsal Amygdala is Sensitive to Threat-Related Faces}

Within the EAc, threat-related faces were associated with significant activation of the dorsal amygdala, bilaterally $(p<.05$, FWE-corrected; Left: $t=12.59$, volume $=1,032 \mathrm{~mm}^{3} ; x=-20, y=-10, z=-14$; Right: $t=12.22$, volume $=1,368 \mathrm{~mm}^{3} ; x=22, y=-8, z=-16$; Figure 1a and Supplementary Table 
1), consistent with prior work e.g., ${ }^{16}$. As shown in Supplementary Figure S4, the amygdala cluster overlapped the anatomically defined Ce ROI, with the left and right peaks lying in the dorsocaudal region where the Ce, medial, and basomedial nuclei abut.

On an exploratory basis, we also computed a series of whole-brain analyses. Results indicated that the dorsal amygdala and fusiform cortex ('fusiform face area') were significantly more sensitive to threat-related faces, whereas the parahippocampal cortex ('parahippocampal place area') was significantly more sensitive to places, as expected ${ }^{52,53}(p<.05$, FWE-corrected; Supplementary Figure S5 and Supplementary

Table 2). 
a.

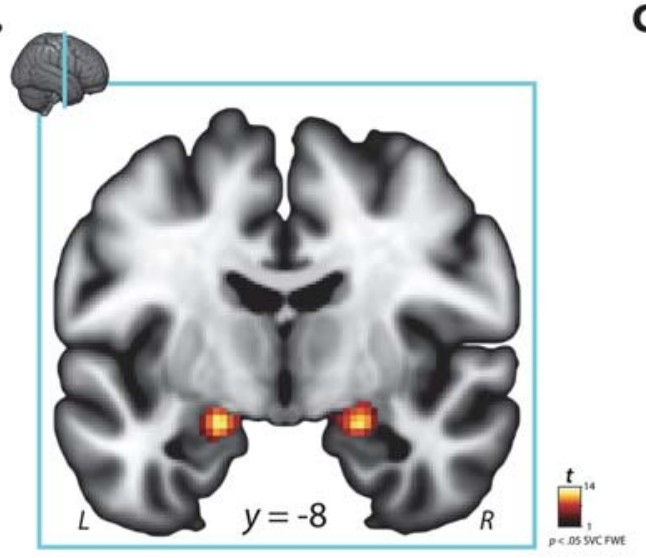

C.

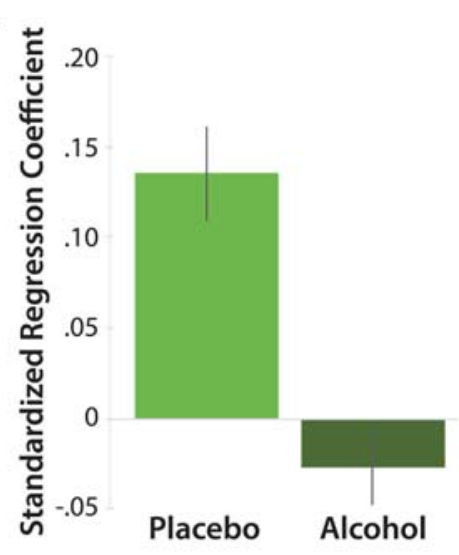

b.

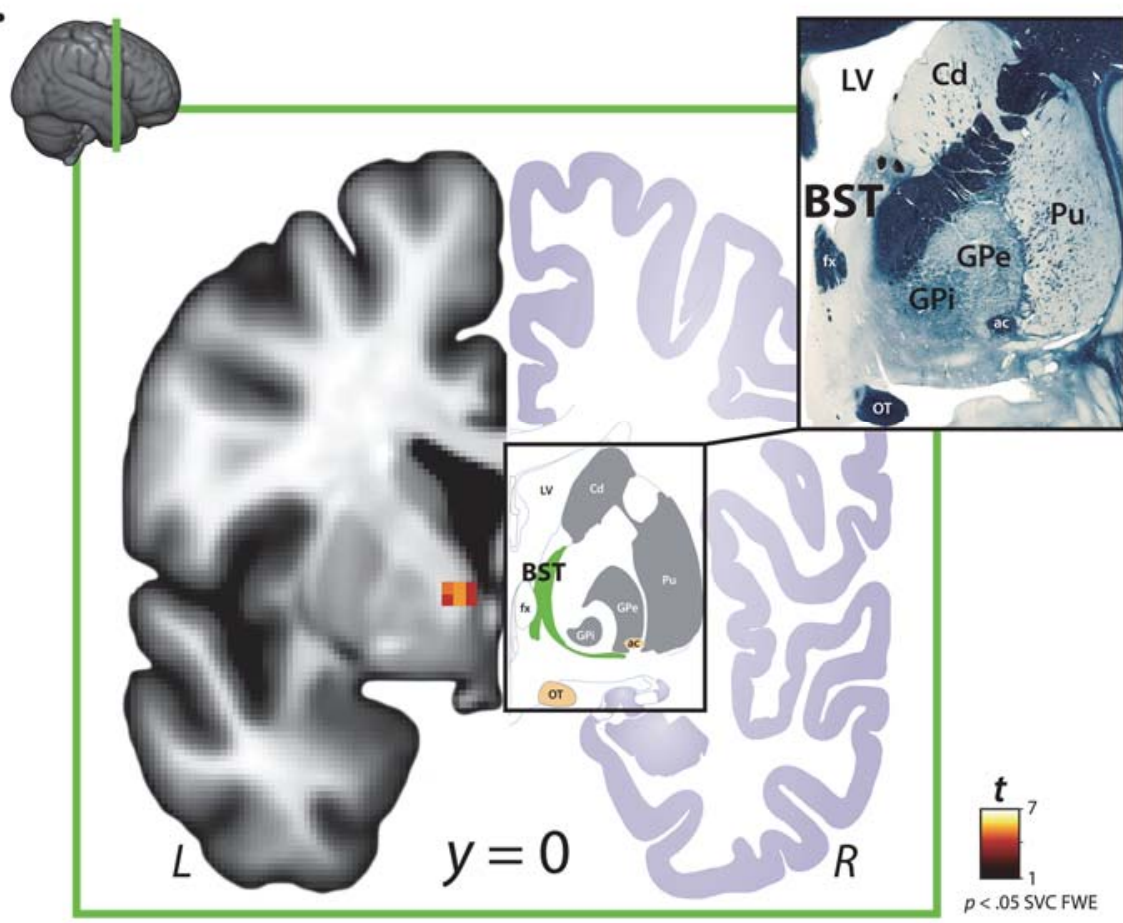

Figure 1. The impact of acute alcohol administration on reactivity to threat-related cues in the central extended amygdala. A. Consistent with prior work, voxelwise regression analyses revealed significant activation to threat-related faces in the dorsal amygdala $(p<.05$, FWE corrected for the volume of the anatomically defined EAc region-of-interest; total volume: 1,205 voxels; $9,640 \mathrm{~mm}^{3}$ ). Inset indicates the location of the coronal slice. Significant clusters within the EAc ROI (Supplementary Figure S2) are depicted here. For additional results, see Supplementary Figures S4 and S5 and Supplementary Tables S1 and S2. B. Voxelwise analyses revealed a significant reduction in reactivity to threat-related faces in the region of the left BST in the alcohol compared to the placebo group (same threshold; equivalent to testing the Group $\times$ Condition interaction). The left half of the panel depicts the BST cluster. The right half depicts the BST (green) in the corresponding section of the atlas of Mai and colleagues (2015). Note the similar appearance of key landmarks, including the fornix and lateral ventricle (white), as well as the optic tract and anterior commissure (gold). Upper left inset indicates the location of the coronal slice. Upper right inset depicts the myeloarchitecture (Weigert fiber stain) of this region in the atlas. The left BST was the only significant cluster in EAc-focused or whole-brain analyses. For additional results, see Supplementary Figure S6 and Supplementary Table S3. C. For illustrative purposes, barplot depicts mean standardized regression coefficients extracted from the peak voxel in the BST cluster for the alcohol (light green) and placebo (dark green) groups. Hypothesis testing was performed on a voxelwise basis (corrected for multiple comparisons). Error bars indicate the standard error of the mean. Portions of this figure were adapted with permission from the atlas of Mai and colleagues ${ }^{54}$. Abbreviationsac, anterior commissure; BST, bed nucleus of the stria terminalis; Cd, caudate; EAc, central division of the extended amygdala; FWE, family-wise error; fx, fornix; GPe, external globus pallidus; GPi, internal globus pallidus; L, left hemisphere; LV, lateral ventricle; OT, optic tract; $\mathrm{Pu}$, putamen; $\mathrm{R}$, right hemisphere; SVC, small volume correction. 


\section{Alcohol Dampens BST Reactivity to Threat-Related Faces}

Within the EAc, acute alcohol administration was associated with a significant reduction in left BST reactivity to threat-related faces (Group $\times$ Condition: $p<.05$, FWE-corrected; $t=5.46$, volume $=104 \mathrm{~mm}^{3} ; x=-8, y=-2, z=0$; Figures 1b-1c and Supplementary Table 3). As shown in Supplementary Figure S6, the left BST cluster overlapped the anatomically defined BST ROI. Exploratory whole-brain analyses revealed no additional clusters. As detailed in the Supplement, control analyses indicated that the dampening effects of alcohol on BST reactivity to threatrelated faces were not a consequence of group differences in performance.

\section{Alcohol Exerts Similar Effects in the Ce and the BST}

To assess regional differences in EAc activation in an unbiased manner ${ }^{21}$, standardized contrast coefficients (i.e., faces vs. places) were extracted from the left and right Ce and BST — the two major subdivisions of the EAc-using anatomically defined, a priori ROIs, as shown in the upper portion of Figure 2 (Ce: cyan; BST: green). A mixed-model GLM was then used to compare the impact of Group and Hemisphere on regional reactivity to threat-related faces. Analyses revealed greater threat-related activation in the Ce relative to the BST (Region: $F(1,47)=32.99, p<.001$ ) and a near-significant alcohol-dampening effect across regions (Group: $\left.F_{W-s}(1,47)=3.93, p=.053\right]$. Other omnibus effects were not significant ( $p s>15)$. Analyses performed using a performance-matched sub-sample revealed similar results (Supplement). Collectively, these observations indicate that alcohol acutely dampens reactivity to threat-related faces and it does so similarly in the Ce and the BST. 


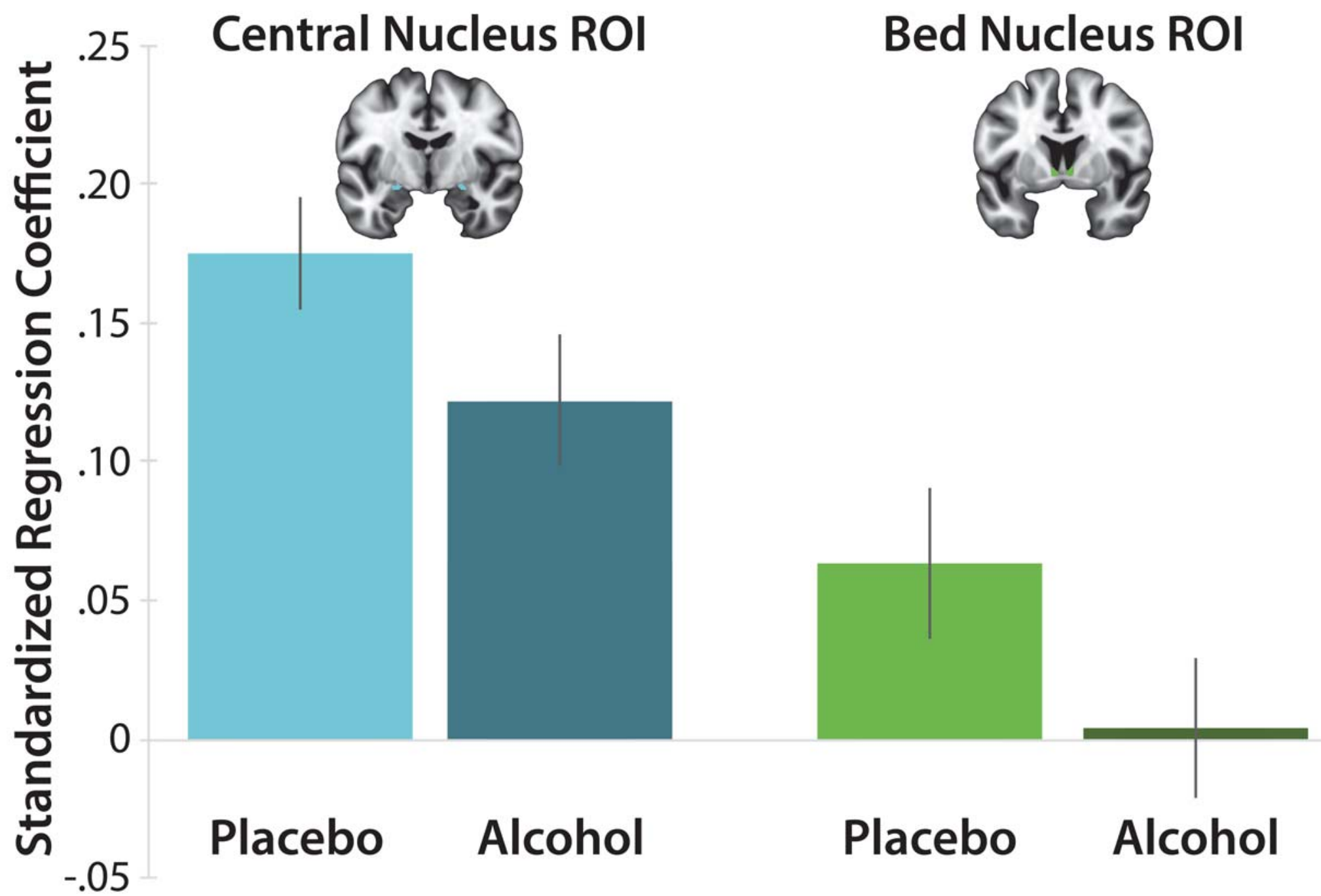

Figure 2. The impact of acute alcohol administration on the two major divisions of the EAc. Barplot depicts mean regression coefficients associated with the emotionalfaces/places task for the anatomically defined Ce and BST ROIs for each group. The Ce was significantly more reactive to threat-related faces, relative to the BST ( $p<.001$ ). On average, subjects randomly assigned to the alcohol group showed significantly less reactivity to threat-related faces, relative to those in the placebo group ( $p=.053$; equivalent to testing the Group $\times$ Condition interaction). The Group $\times$ Region interaction was not significant $(p=.88)$, suggesting that the Ce and BST are similarly sensitive to the threat-dampening impact of acute alcohol administration. Error bars indicate the standard error of the mean. Abbreviations-EAc, central extended amygdala; ROI, region of interest. 


\section{DISCUSSION}

Recent epidemiological work indicates that "the United States is facing a crisis with alcohol use, one that is currently costly and about to get worse" 55, yet the neural circuitry most relevant to human alcohol consumption has remained incompletely understood. The etiology of alcohol abuse is complex and involves multiple neurocognitive and motivational systems ${ }^{5}$, but observations gleaned from clinical research in humans and mechanistic work in rodents highlights the potential importance of negative reinforcement effects mediated by the EAc 5,8. Leveraging a placebo-controlled randomized-groups design, the present results demonstrate for the first time that that acute alcohol administration significantly dampens reactivity to threat-related faces in the BST (Figure 1). Analyses performed using unbiased, anatomically defined ROIs revealed a similar pattern of alcohol dampening across the Ce and BST (Figure 2). Control analyses indicated that these results were not an artifact of group differences in performance (Supplement). Collectively, these findings indicate that acute alcohol intoxication dampens reactivity to threat-related faces in humans and it does so similarly across the EAc.

The present results reinforce the translational relevance of addiction models derived from preclinical research in mice and rats 5,9 . Work in rodent models directly implicates the EAc in the threat-dampening consequences of alcohol 7,56-59. Immediate early gene studies show that alcohol robustly engages both the Ce and the BST ${ }^{60}$. While the molecular consequences of alcohol are complex, acute alcohol inhibits excitatory (i.e., glutamatergic) neurotransmission in the Ce and the BST 61-63 and increases the release of the inhibitory neurotransmitter gammaaminobutyric acid (GABA) in the Ce via interactions with the corticotropin-releasing factor (CRF) type-1 receptor 62,64-69. Enhanced GABAergic tone within the Ce is, in turn, thought to inhibit cells in the BST and other downstream effector regions 58,65 . Other work indicates that CRF projections from the Ce to the BST play a critical role in excessive drinking in alcohol-dependent rats ${ }^{70}$, consistent with evidence implicating 
the EAc in withdrawal-related negative affect and stress-induced substance use ${ }^{31}$. While our observations align with this body of research, as with any human neuroimaging study, our conclusions are tempered by questions about the origins and significance of the blood oxygen leveldependent fMRI signal 71.

The present results are broadly consistent with clinical research underscoring the importance of negative reinforcement mechanisms in recreational drinking as well as alcohol abuse. Many drinkers expect alcohol to reduce tension or stress ${ }^{72}$ and those seeking stress reduction are at greater risk for developing an AUD 73,74. Individuals with a more anxious temperament and patients with anxiety orders (e.g., social phobia) are more likely to misuse alcohol 75,76, and homologous effects have been found in rodents ${ }^{6,77}$. In the laboratory, moderate doses of alcohol selectively dampen defensive responses (e.g., startle) elicited by uncertain physical danger ${ }^{8}$. Our results suggest that some of these effects may reflect a downstream consequence of dampened EAc reactivity to potential threat.

Although static images of fearful faces do not elicit robust signs of fear or anxiety ${ }^{78}$, they are relevant to daily experience, can increase anxiety symptoms, and are perceived as more threatening and arousing than neutral or happy faces $79-81$. Fearful faces have also been shown to promote vigilance, increasing visual sensitivity, boosting the resolution of visual processing, and enhancing the efficiency of attentional search 16. Vigilance is thought to be mediated by circuits centered on the EAc and, once elicited, increases the likelihood experiencing more extreme or pervasive states of anxiety 16,82 . A key challenge for future research will be to examine the impact of alcohol on EAc reactivity to more intense anxiety-provoking stimuli, such as uncertain threat-of-shock. This approach would dovetail with work in rodent models, enhancing the likelihood of successful bi-directional translation ${ }^{34}$. Prospective longitudinal imaging research would be useful for understanding the 
relevance of EAc circuitry to the development of alcohol use disorder and other addictions. Combined with more naturalistic measures of stress-induced drinking in the laboratory or field (e.g., ecological momentary assessment), this approach might provide a means of stratifying at-risk populations or patients into the subset for whom negative reinforcement circuits are most relevant to intervention.

The present results are also relevant to on-going debates about the functional organization of the EAc ${ }^{13}$. Among researchers focused on humans, it is widely believed that the Ce and BST are functionally dissociable. Inspired by an earlier generation of lesion and inactivation studies in rodents ${ }^{83}$, this 'double-dissociation' or 'strict-segregation' hypothesis suggests that the Ce (or the amygdala more generally) rapidly assembles phasic responses to clear-and-immediate threats (e.g., a cue associated with the imminent delivery of shock), whereas the BST comes on-line more slowly and is responsible for orchestrating sustained responses to dangers that are diffuse, uncertain, or remote. While this hypothesis remains popular, and has even been incorporated into the National Institute of Mental Health's Research Domain Criteria (RDoC) initiative, a range of evidence gleaned from studies of rodents, monkeys, and humans makes it clear that while the Ce and the BST are certainly not interchangeable, they are more alike than different 12,84 . Leveraging an unbiased ROI approach, our results extend this work to show that the Ce and BST are similarly sensitive to the threat-dampening effects of alcohol. Whether this generalizes to more intense threatrelated cues (e.g., film clips) or other anxiolytics (e.g., benzodiazepines) remains an important avenue for future studies.

Existing treatments for alcohol use and other additions are far from curative 4,85 , highlighting the need to develop a deeper understanding of the underlying motivational processes and neural systems. The present results demonstrate that acute alcohol intoxication dampens reactivity to threat-related cues across the human EAc. The use of a relatively large sample (Table 1), placebo-controlled between-groups design, 
ecologically relevant dosing, fMRI-optimized task, best practices for the acquisition and processing of functional neuroimaging data, and unbiased ROI analytic approach enhances confidence in the clinical and translational significance of these results. More broadly, these findings set the stage for mechanistic work aimed at developing more effective treatments for alcohol abuse and other debilitating addictions. 


\section{FIGURES AND LEGENDS}

a.

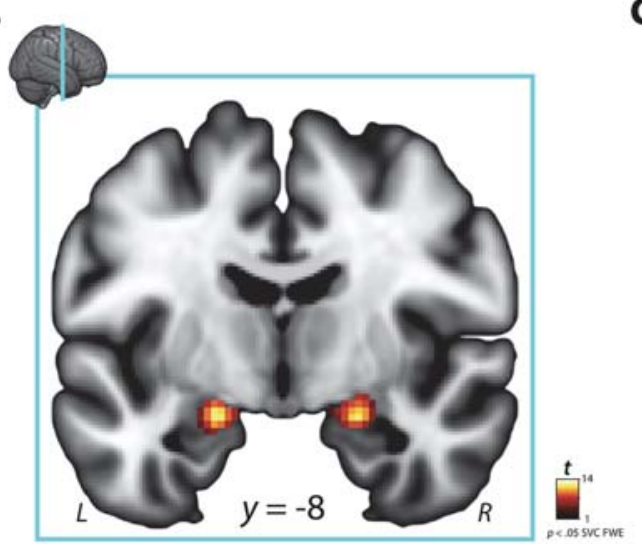

C.

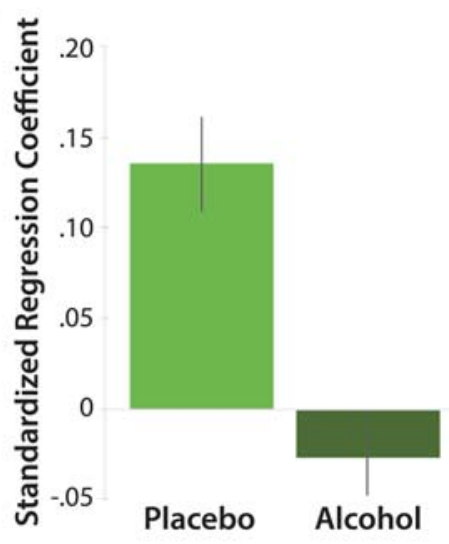

b.

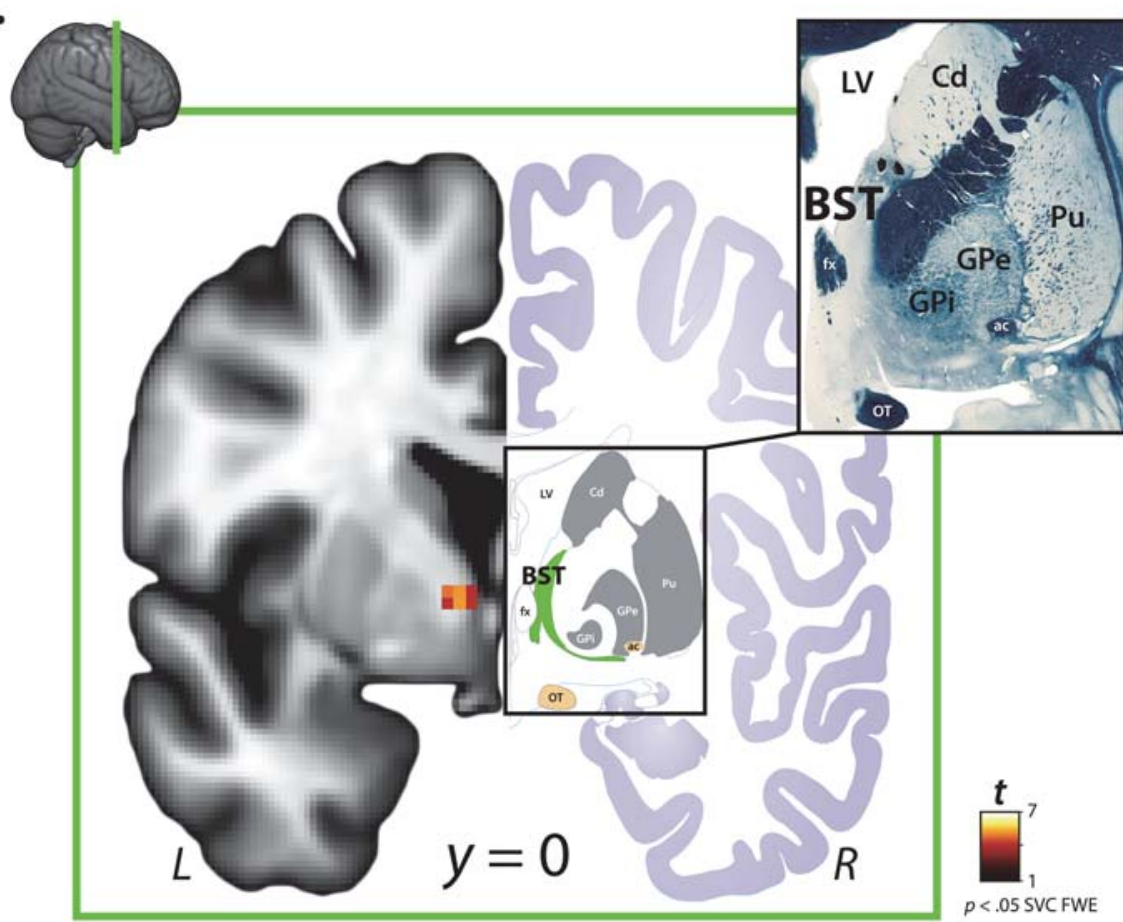

Figure 1. The impact of acute alcohol administration on reactivity to threat-related cues in the central extended amygdala. A. Consistent with prior work, voxelwise regression analyses revealed significant activation to threat-related faces in the dorsal amygdala $(p<.05$, FWE corrected for the volume of the anatomically defined EAc region-of-interest; total volume: 1,205 voxels; $9,640 \mathrm{~mm}^{3}$ ). Inset indicates the location of the coronal slice. Significant clusters within the EAc ROI (Supplementary Figure S2) are depicted here. For additional results, see Supplementary Figures S4 and S5 and Supplementary Tables S1 and S2. B. Voxelwise analyses revealed a significant reduction in reactivity to threat-related faces in the region of the left BST in the alcohol compared to the placebo group (same threshold; equivalent to testing the Group $\times$ Condition interaction). The left half of the panel depicts the BST cluster. The right half depicts the BST (green) in the corresponding section of the atlas of Mai and colleagues (2015). Note the similar appearance of key landmarks, including the fornix and lateral ventricle (white), as well as the optic tract and anterior commissure (gold). Upper left inset indicates the location of the coronal slice. Upper right inset depicts the myeloarchitecture (Weigert fiber stain) of this region in the atlas. The left BST was the only significant cluster in EAc-focused or whole-brain analyses. For additional results, see Supplementary Figure S6 and Supplementary Table S3. C. For illustrative purposes, barplot depicts mean standardized regression coefficients extracted from the peak voxel in the BST cluster for the alcohol (light green) and placebo (dark green) groups. Hypothesis testing was performed on a voxelwise basis (corrected for multiple comparisons). Error bars indicate the standard error of the mean. Portions of this figure were adapted with permission from the atlas of Mai and colleagues ${ }^{54}$. Abbreviationsac, anterior commissure; BST, bed nucleus of the stria terminalis; Cd, caudate; EAc, central division of the extended amygdala; FWE, family-wise error; fx, fornix; GPe, external globus pallidus; GPi, internal globus pallidus; L, left hemisphere; LV, lateral ventricle; $\mathrm{OT}$, optic tract; $\mathrm{Pu}$, putamen; $\mathrm{R}$, right hemisphere; SVC, small volume correction. 


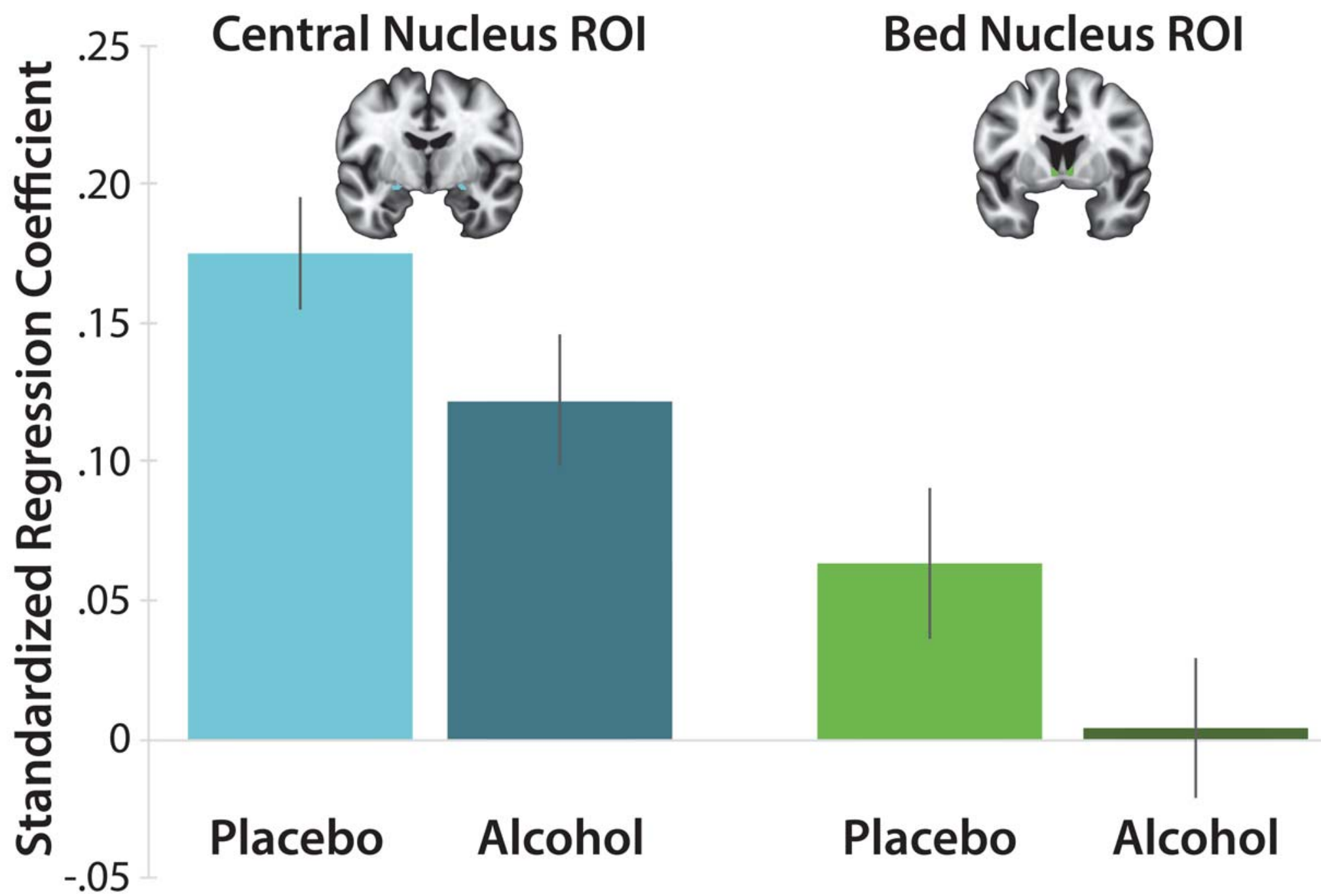

Figure 2. The impact of acute alcohol administration on the two major divisions of the EAc. Barplot depicts mean regression coefficients associated with the emotionalfaces/places task for the anatomically defined Ce and BST ROIs for each group. The Ce was significantly more reactive to threat-related faces, relative to the BST ( $p<.001$ ). On average, subjects randomly assigned to the alcohol group showed significantly less reactivity to threat-related faces, relative to those in the placebo group ( $p=.053$; equivalent to testing the Group $\times$ Condition interaction). The Group $\times$ Region interaction was not significant $(p=.88)$, suggesting that the Ce and BST are similarly sensitive to the threat-dampening impact of acute alcohol administration. Error bars indicate the standard error of the mean. Abbreviations-EAc, central extended amygdala; ROI, region of interest. 


\section{TABLES}

Table 1. The effects of acute alcohol administration on reactivity to threat-related faces in human imaging studies.

\begin{tabular}{|c|c|c|c|c|c|}
\hline Citation & $\begin{array}{l}N(\% \\
\text { Male) }\end{array}$ & $\begin{array}{l}\text { Headcoil } \\
\text { (Channels) }\end{array}$ & $\begin{array}{l}\text { Native EPI } \\
\text { Resolution } \\
(\mathrm{mm})\end{array}$ & $\begin{array}{l}\text { Smoothing } \\
(\mathrm{mm})\end{array}$ & Normalization ${ }^{a}$ \\
\hline & $\begin{array}{l}49 \\
(53 \%): \\
27\end{array}$ & & & & \\
\hline $\begin{array}{l}\text { Present } \\
\text { study }\end{array}$ & $\begin{array}{l}\text { alcohol, } \\
22 \\
\text { placebo }\end{array}$ & 32 & $2 \times 2 \times 2$ & 6 & $\begin{array}{l}\text { BBR and } \\
\text { ANTS/SyN } \\
\text { diffeomorphic }\end{array}$ \\
\hline
\end{tabular}

Gilman
et al.,
2008

2008$$
(42 \%)
$$

16

$3.75 \times 3.75$
$\times 5$

$$
\times 5
$$

"AFNI" (affine?)

Design $\quad$ Route
single-
blind,

Mean

blind,

placebo-

controlled,

randomized

groups
double-

blind,

placebo-

controlled,

randomized

cross-over IV

double-

blind,

placebo-

controlled,

randomized

et al., 14

$2012^{\mathrm{b}}$

(100\%) 16

$3.75 \times 3.75$

$\times 5$

6

"AFNI" (affine?)

cross-over

single-

blind,

placebo-

controlled,

Padula
et al.,

BAL
2011

12

(58\%)

8

$3.43 \times 3.43$

$\times 5$

6

randomized

cross-over

double-

blind,

placebo-

controlled

Sripada

et al.,

12

(83\%) ?

$3.75 \times 3.75$

$\times 5$

8

SPM12 (EPI

randomized cross-over
Threshold Task Key Results

.05 corr Threat-

and related

anatomical faces vs.

ROIs in buildings

Ce/BST (blocked)

unclear Threat-

voxelwise related vs.

and

neutral

spherical faces

ROIs in (event-

amygdala related)

.01 corr Threat-

and related vs.

spherical neutral

ROI in faces

right (event-

amygdala

related)

Threat-

related

faces vs.

happy faces

vs. shapes

Oral $\quad 0.07 \quad .05$ unc

(blocked)

N/A

Significant Threat $>$ Neutra类

in right amygdala, NS with o

alcohol; Voxelwise

Treatment $\times$ Condition N\&S

$=.08$ [uncorr] for ROI

analyses)

Significant Threat > Neut

in the right amygdala, $\mathrm{NS}^{+} \mathrm{C}$

with alcohol; Whole-brai $\overline{\mathrm{s}}$

Treatment $\times$ Condition not

reported ( $p=.02$ [uncorr $\$$

ROI analysis)

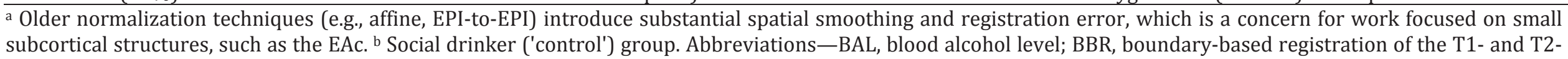
weighted images; Corr, corrected; IV, intravenous; N/A, not applicable; NS, not significant; Unc, uncorrected. 
Table 2. Demographic and descriptive variables.

\begin{tabular}{|c|c|c|c|c|}
\hline & Total & Placebo & Alcohol & Difference \\
\hline Sample size & 49 & 22 & 27 & $\mathrm{~N} / \mathrm{A}$ \\
\hline Mean Age in Years $(S D)$ & $22.4(2.5)$ & $22.1(1.4)$ & $22.6(3.1)$ & $t(47)=.69, p=0.50$ \\
\hline Gender: Female/Male & $23 / 26$ & $11 / 11$ & $12 / 15$ & $\chi^{2}=.15, p=0.8$ \\
\hline Mean BALa $(S D)$ & $\mathrm{N} / \mathrm{A}$ & $0.00(0.00)$ & $0.09(0.02)$ & $t(47)=27.20, p<.001$ \\
\hline $\begin{array}{l}\text { Mean Estimated Number of } \\
\text { Drinks Consumed During the } \\
\text { Study }(S D)\end{array}$ & $\mathrm{N} / \mathrm{A}$ & $2.07(1.09)^{\mathrm{b}}$ & $4.56(1.25)^{c}$ & $t(47)=7.32, p<.001$ \\
\hline $\begin{array}{l}\text { Mean Motion, Frame-to- } \\
\text { Frame Displacement }(S D)\end{array}$ & $.13(.03)$ & $.12(.03)$ & $.13(.03)$ & $t(47)=1.01, p=.32$ \\
\hline
\end{tabular}

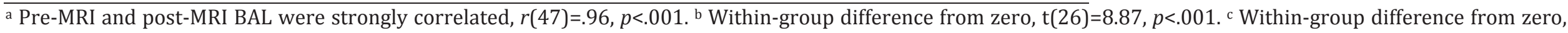
$t(26)=18.93, p<.001$. 


\section{CONTRIBUTIONS}

C.M.K, A.J.S., and J.F.S. designed the imaging study based on procedures originally developed and refined by D.E.B. and J.J.C. for psychophysiological research. C.M.K. and J.F.S. collected data. J.H., C.M.K., and J.F.S. processed data. J.H., C.M.K., A.J.S., and J.F.S. analyzed data. J.H., A.J.S., A.S.F., and J.F.S. interpreted data. J.H., C.M.K., J.F.S., and A.J.S. wrote the paper. A.J.S. created figures. J.H., J.F.S., and A.J.S. created tables. A.S.F., D.E.B., and J.J.C. provided theoretical guidance. A.J.S. funded and supervised all aspects of the study. All authors contributed to reviewing and revising the paper and approved the final version.

\section{ACKNOWLEDGEMENTS}

Authors acknowledge critical feedback from E. Bernat, L. Pessoa, and M. Roesch and assistance from K. DeYoung, L. Friedman, M. Gamer, B. Nacewicz, M. Stockbridge, S. Padmala, P. Spechler, R. Tillman, and personnel from the Affective and Translational Neuroscience Laboratory and Maryland Neuroimaging Center. This work was supported by the University of California, Davis; University of Maryland, College Park; University of Wisconsin-Madison; and National Institutes of Health (DA040717 and MH107444). Development of the NimStim Face Database was overseen by N. Tottenham and supported by the MacArthur Foundation. Authors declare no conflicts of interest.

\section{DATA AVAILABILITY/SHARING}

All of the key statistical maps and regions-of-interest have been or will be uploaded to NeuroVault.org. 


\section{REFERENCES}

1 Grant, B. F. et al. Prevalence of 12-month alcohol use, high-risk drinking, and DSM-IV alcohol use disorder in the United States, 20012002 to 2012-2013: Results from the National Epidemiologic Survey on Alcohol and Related Conditions. JAMA psychiatry 74, 911-923 (2017).

Forouzanfar, M. H. et al. Global, regional, and national comparative risk assessment of 79 behavioural, environmental and occupational, and metabolic risks or clusters of risks, 1990-2015: a systematic analysis for the Global Burden of Disease Study 2015. The Lancet 388, 1659-1724 (2016).

Rehm, J. et al. Global burden of disease and injury and economic cost attributable to alcohol use and alcohol-use disorders. The Lancet 373, 2223-2233 (2009).

Willenbring, M. L. Gaps in clinical prevention and treatment for alcohol use disorders: Costs, consequences, and strategies. Alcohol research: current reviews 35, 238 (2014). Medicine 374, 363-371 (2016). Studies on Alcohol, Supplement, 9-19 (1993). 
Kaye, J. T., Bradford, D. E., Magruder, K. P. \& Curtin, J. J. Probing for neuroadaptations to unpredictable stressors in addiction:

Translational methods and emerging evidence. Journal of Studies on Alcohol and Drugs 78, 353-371 (2017).

Wise, R. A. \& Koob, G. F. The development and maintenance of drug addiction. Neuropsychopharmacology 39, 254 (2014).

Avery, S., Clauss, J. \& Blackford, J. The human BNST: functional role in anxiety and addiction. Neuropsychopharmacology 41, 126-141 (2016). 34-38 (2011).

Fox, A. S. \& Shackman, A. J. The central extended amygdala in fear and anxiety: Closing the gap between mechanistic and neuroimaging research. Neuroscience letters (in press).

Shackman, A. J. \& Fox, A. S. Contributions of the central extended amygdala to fear and anxiety. Journal of Neuroscience 36, 8050-8063 (2016).

14 Davis, M. \& Whalen, P. J. The amygdala: vigilance and emotion. Mol Psychiatry 6, 13-34 (2001).

Fox, A. S., Oler, J. A., Tromp, D. P., Fudge, J. L. \& Kalin, N. H. Extending the amygdala in theories of threat processing. Trends in neurosciences $\mathbf{3 8}, 319-329$ (2015).

Shackman, A. J. et al. The neurobiology of dispositional negativity and attentional biases to threat: implications for understanding anxiety disorders in adults and youth. Journal of experimental psychopathology 7, 311 (2016). 
Del-Ben, C. M. et al. Effects of diazepam on BOLD activation during the processing of aversive faces. Journal of Psychopharmacology 26, 443-451 (2012).

Paulus, M. P., Feinstein, J. S., Castillo, G., Simmons, A. N. \& Stein, M. B. Dose-dependent decrease of activation in bilateral amygdala and insula by lorazepam during emotion processing. Archives of General Psychiatry 62, 282-288 (2005).

Eklund, A., Nichols, T. E. \& Knutsson, H. Cluster failure: why fMRI inferences for spatial extent have inflated false-positive rates. Proceedings of the National Academy of Sciences 113, 7900-7905 (2016).

Poldrack, R. A. et al. Scanning the horizon: towards transparent and reproducible neuroimaging research. Nature Reviews

Neuroscience 18, 115-126 (2017).

Fox, A. S., Lapate, R. C., Davidson, R. J. \& Shackman, A. J. in The nature of emotion. Fundamental questions (2nd ed.) (eds A. S. Fox, R. C. Lapate, A. J. Shackman, \& R. J. Davidson) ( Oxford University Press, 2018).

Gilman, J. M., Ramchandani, V. A., Crouss, T. \& Hommer, D. W. Subjective and neural responses to intravenous alcohol in young adults with light and heavy drinking patterns. Neuropsychopharmacology 37, 467-477 (2012).

Gilman, J. M., Ramchandani, V. A., Davis, M. B., Bjork, J. M. \& Hommer, D. W. Why we like to drink: a functional magnetic resonance imaging study of the rewarding and anxiolytic effects of alcohol. Journal of Neuroscience 28, 4583-4591 (2008). Alcohol and alcoholism 46, 547-552 (2011). 
Cannon, T. D., Cao, H., Mathalon, D. H. \& Gee, D. G. Reliability of an fMRI paradigm for emotional processing in a multisite longitudinal study: Clarification and implications for statistical power. Human brain mapping 39, 599-601 (2018).

Smith, J. F., Hur, J., Kaplan, C. M. \& Shackman, A. J. The impact of spatial normalization for functional magnetic resonance imaging data analyses revisited. bioRxiv (2018).

Theiss, J. D., Ridgewell, C., McHugo, M., Heckers, S. \& Blackford, J. U. Manual segmentation of the human bed nucleus of the stria terminalis using 3T MRI. Neuroimage 146, 288-292, doi:10.1016/j.neuroimage.2016.11.047 (2016).

Tillman, R. M. et al. Intrinsic functional connectivity of the central extended amygdala. Human brain mapping 39, 1291-1312 (2018). 491 (2017).

Klumpers, F., Kroes, M. C., Baas, J. M. \& Fernández, G. How human amygdala and bed nucleus of the stria terminalis may drive distinct defensive responses. Journal of neuroscience 37, 9645-9656 (2017). path for animal models. Neuropsychopharmacology 34, 74 (2009).

\section{Bradford, D. E., Shapiro, B. L. \& Curtin, J. J. How bad could it be? Alcohol dampens stress responses to threat of uncertain intensity.}

Psychol Sci 24, 2541-2549, doi:10.1177/0956797613499923 (2013). 
Shokri-Kojori, E., Tomasi, D., Wiers, C. E., Wang, G.-J. \& Volkow, N. D. Alcohol affects brain functional connectivity and its coupling with behavior: greater effects in male heavy drinkers. Molecular psychiatry 22, 1185 (2017).

Curtin, J. J. \& Fairchild, B. A. Alcohol and cognitive control: implications for regulation of behavior during response conflict. Journal of abnormal psychology 112, 424 (2003).

Stout, D. M., Shackman, A. J., Pedersen, W. S., Miskovich, T. A. \& Larson, C. L. Neural circuitry governing anxious individuals' misallocation of working memory to threat. Scientific reports 7, 8742 (2017).

Swartz, J. R., Knodt, A. R., Radtke, S. R. \& Hariri, A. R. A neural biomarker of psychological vulnerability to future life stress. Neuron 85 505-511 (2015).

Maus, B., van Breukelen, G. J., Goebel, R. \& Berger, M. P. Optimization of blocked designs in fMRI studies. Psychometrika 75, 373-390 (2010). (2007).

Plichta, M. M. et al. Amygdala habituation: a reliable fMRI phenotype. NeuroImage 103, 383-390 (2014).

\section{Gamer, M., Schmitz, A. K., Tittgemeyer, M. \& Schilbach, L. The human amygdala drives reflexive orienting towards facial features.} Current Biology 23, R917-R918 (2013). 
Avants, B. B. et al. A reproducible evaluation of ANTs similarity metric performance in brain image registration. Neuroimage 54, 2033-2044, doi:10.1016/j.neuroimage.2010.09.025 (2011).

Cox, R. W. AFNI: Software for analysis and visualization of functional magnetic resonance neuroimages. Computers and Biomedical Research 29, 162-173 (1996).

O'Craven, K. M. \& Kanwisher, N. Mental imagery of faces and places activates corresponding stimulus-specific brain regions. Journal of cognitive neuroscience 12, 1013-1023 (2000).

Behzadi, Y., Restom, K., Liau, J. \& Liu, T. T. A component based noise correction method (CompCor) for BOLD and perfusion based fMRI. Neuroimage 37, 90-101 (2007).

Yilmazer-Hanke, D. M. in The human nervous system (eds J. K. Mai \& G. Paxinos) 759-834 (Academic Press, 2012).

Trim, R. S. et al. Acute Ethanol Effects on Brain Activation in Low-and High-Level Responders to Alcohol. Alcoholism: clinical and experimental research 34, 1162-1170 (2010).

Kanwisher, N. Functional specificity in the human brain: a window into the functional architecture of the mind. Proceedings of the National Academy of Sciences 107, 11163-11170 (2010). Sabatinelli, D. et al. Emotional perception: meta-analyses of face and natural scene processing. Neuroimage 54, 2524-2533 (2011). Mai, J. K., Majtanik, M. \& Paxinos, G. Atlas of the human brain. (Academic Press, 2015). Schuckit, M. A. Remarkable Increases in Alcohol Use Disorders. JAMA psychiatry 74, 869-870 (2017).

Gilpin, N. W., Herman, M. A. \& Roberto, M. The central amygdala as an integrative hub for anxiety and alcohol use disorders. Biological psychiatry 77, 859-869 (2015). 
Lovinger, D. M. \& Kash, T. L. Mechanisms of neuroplasticity and ethanol's effects on plasticity in the striatum and bed nucleus of the stria terminalis. Alcohol research: current reviews 37, 109 (2015).

Roberto, M., Gilpin, N. W. \& Siggins, G. R. The central amygdala and alcohol: role of $\gamma$-aminobutyric acid, glutamate, and neuropeptides. Cold Spring Harbor perspectives in medicine 2, a012195 (2012).

Silberman, Y. \& Winder, D. G. Ethanol and corticotropin releasing factor receptor modulation of central amygdala neurocircuitry: an update and future directions. Alcohol 49, 179-184 (2015).

Leriche, M., Mendez, M., Zimmer, L. \& Berod, A. Acute ethanol induces Fos in GABAergic and non-GABAergic forebrain neurons: a double-labeling study in the medial prefrontal cortex and extended amygdala. Neuroscience 153, 259-267 (2008).

Kash, T. L., Matthews, R. T. \& Winder, D. G. Alcohol inhibits NR2B-containing NMDA receptors in the ventral bed nucleus of the stria terminalis. Neuropsychopharmacology 33, 1379-1390 (2008).

Roberto, M., Madamba, S. G., Stouffer, D. G., Parsons, L. H. \& Siggins, G. R. Increased GABA release in the central amygdala of ethanoldependent rats. Journal of Neuroscience 24, 10159-10166 (2004).

Weitlauf, C., Egli, R. E., Grueter, B. A. \& Winder, D. G. High-frequency stimulation induces ethanol-sensitive long-term potentiation at glutamatergic synapses in the dorsolateral bed nucleus of the stria terminalis. Journal of Neuroscience 24, 5741-5747 (2004). release in central amygdala. Proceedings of the National Academy of Sciences 105, 8410-8415 (2008). 
Nie, Z., Madamba, S. G. \& Siggins, G. R. Ethanol enhances $\gamma$-aminobutyric acid responses in a subpopulation of nucleus accumbens neurons: role of metabotropic glutamate receptors. Journal of Pharmacology and Experimental Therapeutics 293, 654-661 (2000). Roberto, M. et al. Corticotropin releasing factor-induced amygdala gamma-aminobutyric acid release plays a key role in alcohol dependence. Biological psychiatry 67, 831-839 (2010). Roberto, M., Madamba, S. G., Moore, S. D., Tallent, M. K. \& Siggins, G. R. Ethanol increases GABAergic transmission at both pre-and postsynaptic sites in rat central amygdala neurons. Proceedings of the National Academy of Sciences 100, 2053-2058 (2003). Varodayan, F., Logrip, M. \& Roberto, M. P/Q-type voltage-gated calcium channels mediate the ethanol and CRF sensitivity of central amygdala GABAergic synapses. Neuropharmacology 125, 197-206 (2017). de Guglielmo, G. et al. Optogenetic characterization of CeA CRF pathways in alcohol dependence. bioRxiv, 134759 (2017). Logothetis, N. K. What we can do and what we cannot do with fMRI. Nature 453, 869-878 (2008).

Sher, K. J. in Psychological theories of drinking and alcoholism (eds H. T. Blane \& K. E. Leonard) 227-271 (Guilford Press, 1987). alcohol use. Journal of personality and social psychology 69, 990 (1995). collection. Social science \& medicine 65, 2588-2602 (2007). Comorbidity Survey. Archives of general psychiatry 54, 313-321 (1997). 
Moonat, S., Sakharkar, A. J., Zhang, H. \& Pandey, S. C. The role of amygdaloid brain-derived neurotrophic factor, activity-regulated cytoskeleton-associated protein and dendritic spines in anxiety and alcoholism. Addiction biology 16, 238-250 (2011).

Shackman, A. J. et al. Anxiety selectively disrupts visuospatial working memory. Emotion 6, 40 (2006). (2011).

Wieser, M. J. \& Keil, A. Fearful faces heighten the cortical representation of contextual threat. NeuroImage 86, 317-325 (2014).

Grupe, D. W. \& Nitschke, J. B. Uncertainty and anticipation in anxiety: an integrated neurobiological and psychological perspective. Nature Reviews Neuroscience 14, 488 (2013).

Davis, M. Neural systems involved in fear and anxiety measured with fear-potentiated startle. American Psychologist 61, 741 (2006). of the stria terminalis. Journal of Neuroscience 36, 8038-8049 (2016). 